Journal of Behavioral Decision Making, Vol. 10, 53-64 (1997)

\title{
Original and Cumulative Prospect Theory: A Discussion of Empirical Differences
}

\author{
HEIN FENNEMA \\ University of Nijmegen, The Netherlands \\ PETER WAKKER \\ University of Leiden, The Netherlands
}

\begin{abstract}
This paper discusses differences between prospect theory and cumulative prospect theory. It shows that cumulative prospect theory is not merely a formal correction of some theoretical problems in prospect theory, but it also gives different predictions. Some experiments by Lola Lopes are re-analyzed, and are demonstrated to favor cumulative prospect theory over prospect theory. It turns out that the mathematical form of cumulative prospect theory is well suited for modeling the psychological phenomenon of diminishing sensitivity. (C) 1997 by John Wiley \& Sons, Ltd.
\end{abstract}

KEY WORDS prospect theory; diminishing sensitivity; rank-dependence; decision weights; risk aversion

Prospect Theory (PT) has been one of the most important theories of decision making under risk in the past decade, and has been applied in a wide variety of contexts. By including distortions of probabilities, diminishing sensitivity, and the status quo as a reference point, PT can explain the major deviations from expected utility such as the Allais paradox, the certainty effect, and framing effects (Kahneman and Tversky, 1979). However, there are some theoretical problems in PT. The main problem is that the functional form of PT violates 'stochastic dominance' (Kahneman and Tversky, 1979, pp. 283-284). Stochastic dominance requires that a shift of probability mass from bad outcomes to better outcomes leads to an improved prospect.

The theoretical problems have recently been solved in a new version of PT, called cumulative prospect theory (CPT), that was introduced by Tversky and Kahneman (1992); in particular, CPT satisfies stochastic dominance. Similar forms were introduced by Starmer and Sugden (1989) and Luce and Fishburn (1991). Cumulative prospect theory adopts the rank-dependent method for transforming probabilities that was introduced by Quiggin (1982); see also Lopes (1984), Luce (1988), and Allais (1988). For a survey of non-expected utility, see Slovic, Lichtenstein, and Fischhoff (1988) and Camerer (1992).

This paper describes the PT and CPT theories and discusses differences. In particular, we find that CPT does not only avoid some theoretical problems but also gives different empirical predictions that, for the experiments considered in this paper, are better than those of the original PT. The key feature of CPT is that it permits a satisfactory modeling of diminishing sensitivity, not only with respect to 
outcomes but also with respect to changes in probabilities. A major motivation for this paper has been to demonstrate the central role of diminishing sensitivity in human decision making.

The next section describes the theories; empirical predictions are derived subsequently. The presentation requires some mathematical derivations. We hope, however, that these help to clarify the features and the meaning of the new CPT theory. We also hope that this paper can be an aid for making the mathematics of CPT accessible. Because of the empirical importance of CPT, and because its mathematics is not elementary, such an aid is warranted.

\section{THE THEORIES}

This section describes the PT and CPT theories. By $\left(x_{1}, p_{1} ; \ldots ; x_{n}, p_{n}\right)$ we denote the prospect that yields $\$ x_{j}$ with probability $p_{j}, j=1, \ldots, n$. Throughout, for convenience of the exposition, we arrange the prospect so that $x_{1} \leqslant \ldots \leqslant x_{n}$. In PT and CPT, risk attitudes depend both on the attitude towards outcomes (through a value function) and on the attitude towards probabilities (through a weighting function). A risk attitude then becomes a combination of both the attitude towards probability and the attitude towards outcomes.

We first describe PT. In PT, an editing phase is defined in which prospects are organized and reformulated. In the notation of prospects, this implies that same outcomes are to be 'collapsed'. For instance, $(y, 0.3 ; y, 0.2 ; z, 0.5)$ must be rewritten as $(y, 0.5 ; z, 0.5)$. Hence $x_{1}<\ldots<x_{n}$ is assumed in the discussion of PT. Let us emphasize that Kahneman and Tversky (1979) only formulated their theory for prospects with, at most, two nonzero outcomes. We presently extend it in a straightforward manner to prospects with more outcomes, as it was suggested by Kahneman and Tversky (1979, p. 288) and has been used frequently by other authors (e.g. Camerer and Ho, 1994; Schneider and Lopes, 1986; Wakker, 1989). We only give the PT value for prospects $\left(x_{1}, p_{1} ; \ldots ; x_{n}, p_{n}\right)$ with both positive outcomes (gains) and negative outcomes (losses). Then the PT value is

$$
\pi\left(p_{1}\right) v\left(x_{1}\right)+\cdots+\pi\left(p_{n}\right) v\left(x_{n}\right)
$$

Here $v$ is the value function for outcomes, describing the subject's valuation of money. As usual, we set $v(0)=0$ throughout the paper. The probability weighting function $\pi$ describes the subject's attitude towards probabilities. PT adopts a different formula for evaluating prospects with only gains or only losses. As such prospects are not considered in this paper, the formula is not given; for a discussion, see Miyamoto (1987). Kahneman and Tversky (1979) propose a value function with a reflection point at the status quo outcome, located at zero (see Exhibit 1). They find that $v$ is S-shaped, reflecting the principle of 'diminishing sensitivity' for the evaluation of outcomes. For example, the subject discriminates less between 80 and 100 than between 0 and 20, both when these numbers concern gains and when they concern losses. Note that for losses the value function in Exhibit 1 contradicts the common economic assumption of diminishing marginal utility, according to which a dollar is always appreciated less as a person becomes more wealthy. It is also found that $v$ is steeper for losses than for gains ('loss aversion'); e.g. a loss of $\$ 80$ is felt more than a gain of $\$ 80$.

Kahneman and Tversky find that preferences of subjects can best be modeled by a weighting function that enhances small probabilities and reduces higher probabilities. Hence the weighting function is relatively sensitive to changes in probability near the end points 0 and 1 , but is relatively insensitive to changes in probability in the middle region. At the end points the weighting function is not well behaved. Kahneman and Tversky (1979) give a hypothetical weighting function, conforming with their data (see Exhibit 2). The function is curved upwards where it is well-behaved, and this has been the usual assumption in the literature (Camerer, 1989; Starmer, 1992; Lopes, 1993). 


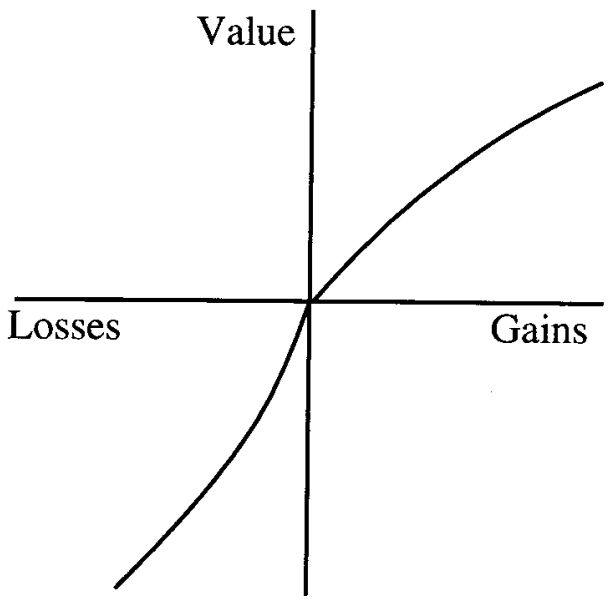

Exhibit 1. The value function assumed by PT and CPT

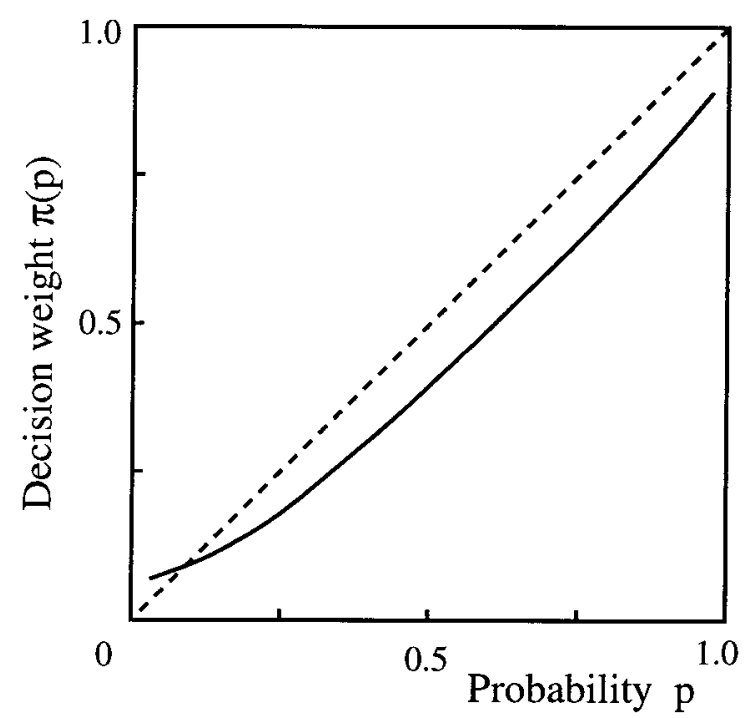

Exhibit 2. A typical weighting function for PT

If the probability weighting function for gains were linear (not curved), then the risk attitude for gains would be entirely determined by the value function, and it is well known that the shape in Exhibit 1 then would imply risk aversion. If the value function were linear for gains, then the risk attitude for gains would be entirely determined by the probability weighting function for gains, and it is well known that the shape in Exhibit 3 implies risk seeking in small-probability prospects and risk aversion in large-probability prospects. In general, the two functions jointly determine the risk attitude.

CPT uses a value function $v$ with the same characteristics as in PT. The determination of decision weights deviates from PT, however. The idea of CPT, elaborated below, is to apply Quiggin's (1982) 'rank-dependent functional' separately to gains and losses, and then take the sum of the two resulting evaluations. A weighting function $w^{+}$is defined for the probabilities associated with gains, and a separate weighting function $w^{-}$is defined for probabilities associated with losses. This allows for different attitudes towards probability for gains than for losses. CPT permits that outcomes are not distinct in the notation $\left(x_{1}, p_{1} ; \ldots ; x_{n}, p_{n}\right)$ for prospects. Suppose for simplicity that $x_{1} \leqslant \ldots \leqslant x_{k} \leqslant 0 \leqslant x_{k+1} \leqslant \ldots \leqslant x_{n}$. Then the CPT value of the prospect $\left(x_{1}, p_{1} ; \ldots ; x_{n}, p_{n}\right)$ is given by the following formula:

$$
\sum_{i=1}^{k} \pi_{i}^{-} v\left(x_{i}\right)+\sum_{i=k+1}^{n} \pi_{i}^{+} v\left(x_{i}\right)
$$

where the decision weights (i.e. the numbers $\pi_{i}^{-}, \pi_{i}^{+}$) are defined by:

$$
\begin{aligned}
& \pi_{1}^{-}=w^{-}\left(p_{1}\right), \quad \pi_{i}^{-}=w^{-}\left(p_{1}+\cdots+p_{i}\right)-w^{-}\left(p_{1}+\cdots+p_{i-1}\right) \quad 2 \leqslant i \leqslant k \\
& \pi_{n}^{+}=w^{+}\left(p_{n}\right), \quad \pi_{i}^{+}=w^{+}\left(p_{i}+\cdots+p_{n}\right)-w^{+}\left(p_{i+1}+\cdots+p_{n}\right) \quad k+1 \leqslant i \leqslant n-1
\end{aligned}
$$

The novelty of this formula as compared to PT is the more subtle way of transforming probabilities. It is instructive to first consider the CPT formula (2) for the special case where $w(p)=p$ for all $p$, 
i.e. probabilities are not transformed. Then the decision weights $\pi_{i}$ are all equal to $p_{i}$, yielding the traditional expected utility formula. This reasoning shows that CPT is indeed a generalization of expected utility.

To explain the above formula, let us first repeat that in PT probabilities for the receipt of separate outcomes were transformed, i.e. each probability $p_{i}$ for receiving the separate outcome $x_{i}$ was transformed into the decision weight $\pi\left(p_{i}\right)$. In the above formula, 'cumulative probabilities' are transformed for gains, and 'decumulative probabilities' for losses. We first consider the case of gains. A cumulative probability describes the probability for receiving an outcome or anything better than that outcome. For instance, $p_{i}+\cdots+p_{n}$ is the cumulative probability of receiving outcome $x_{i}$ or anything better. Decision weights for gains are obtained as differences between transformed values of cumulative probabilities. Similarly, for losses decision weights are obtained as differences between transformed values of consecutive decumulative probabilities, i.e. probabilities describing the receipt of an outcome or anything worse than that outcome.

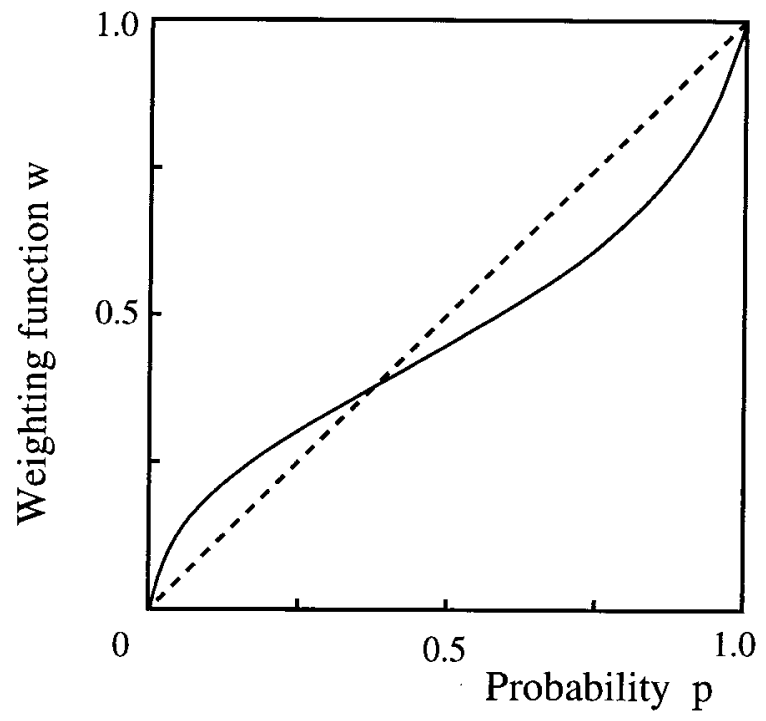

Exhibit 3. A typical CPT weighting function

Tversky and Kahneman (1992) find that for the majority of subjects the weighting function is as depicted in Exhibit 3. This weighting function exhibits 'diminishing sensitivity' with respect to probability changes, i.e. the function is relatively sensitive to changes in probability near the end points 0 and 1 but is relatively insensitive to changes in probability in the middle region. The weighting function in Exhibit 3 is the estimate $w^{+}$for gains; the loss weighting function $w^{-}$has, on average, a similar shape, but it seems to be somewhat higher and less curved.

The problem of the calculation of decision weights in PT, with regard to the generalization to many (different) outcome prospects, can be illustrated by the following example. Suppose we have a prospect with many different outcomes as follows: $(-10,0.05 ; 0,0.05 ; 10,0.05 ; 20,0.05 ; 30,0.05, \ldots$, $180,0.05$ ). If $\pi(0.05)$ is larger than 0.05 (as is commonly found) then each outcome is overweighted, and for the common value functions the prospect will be valued higher than its expected value $\$ 85$ for sure. It is very implausible that people will prefer the prospect to its expected value for sure. This 
anomaly is a consequence of the overweighting of all outcomes, a phenomenon that also underlies the violations of stochastic dominance.

The new way of transforming probabilities circumvents the above problem. Only the extreme outcomes are overweighted. For example, the $\$ 180$ outcome receives decision weight $w^{+}(0.05)$, and the $-\$ 10$ outcome receives decision weight $w^{-}(0.05)$; as Exhibit 3 shows, these outcomes are overweighted. The middle outcomes receive small decision weights; for example, the $\$ 100$ outcome receives a decision weight of $w^{+}(0.45)-w^{+}(0.40)$. Here Exhibit 3 shows that this outcome is underweighted according to CPT. This agrees with a principle of diminishing sensitivity with respect to the impact of outcomes. Intuitively, it seems clear that people will be most sensitive to extreme outcomes and less to intermediate outcomes. The experiments below show that this intuition is correct and also that this can be modeled by CPT and not by (the straightforward generalization of) PT. Arguments for the plausibility of the cumulative form have been given by Wakker (1989). For further elucidations and worked-out numerical examples, see Weber (1994).

The next section describes some empirical differences between PT and CPT. This demonstrates that CPT is not merely a formal correction of some theoretical problems in PT, but that it also gives different predictions. We show that two experiments, conducted by Lopes (1993) to test PT, are especially suited for discriminating between PT and CPT. The first experiment tests the shape of the weighting function. The S-shaped weighting function of CPT explains the data better than the weighting function that is commonly assumed for PT (see Exhibit 2). The first experiment does not reject the general formula of PT, i.e. PT with the S-shaped weighting function of CPT explains the data as well as CPT does. A test of the general formula of PT against that of CPT is provided by the second experiment. PT's transformation of separate probabilities is rejected in favor of CPT's transformation of cumulative probabilities. Let us repeat that Kahneman and Tversky (1979) only formulated their form for prospects with, at most, two nonzero outcomes. The experiments below extend the form to four and six nonzero outcomes.

\section{EXPERIMENT 1}

In the first experiment two prospects are considered, the Bimodal prospect and the Peaked prospect (Exhibit 4). The outcome of each prospect is decided by randomly drawing one ticket from twenty numbered tickets. For example, the Peaked prospect yields $-\$ 200$ if ticket 1 or 2 is drawn, .., $\$ 200$ if ticket 19 or 20 is drawn. Thus,

$$
(-200,0.10 ;-100,0.20 ; 0,0.40 ; 100,0.20 ; 200,0.10)
$$

describes the Peaked prospect. The subjects were undergraduate students, 56 in the original experiment by Lopes and 49 in a replication performed by us. They were asked to state in which of the two

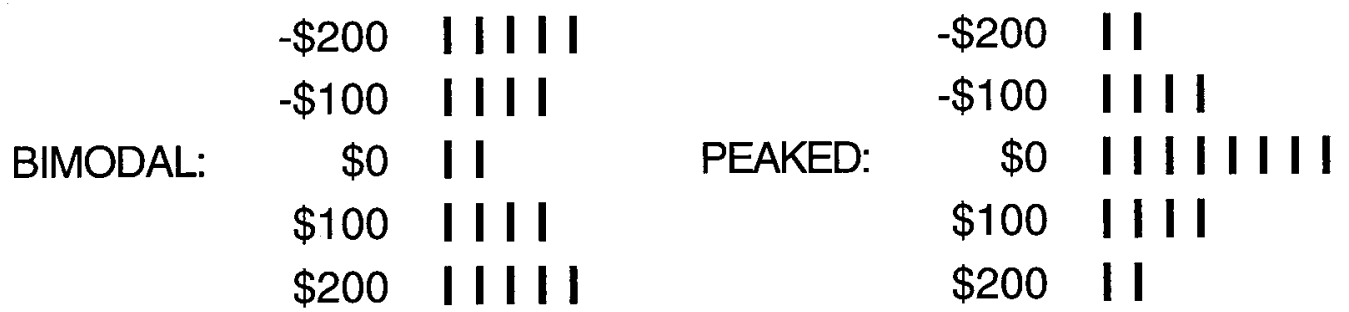

Exhibit 4. Bars indicate lottery tickets, numbered from the upper left to the lower right 
prospects a change of one ticket from a $-\$ 200$ outcome to a $\$ 200$ outcome gives the best improvement. Note that these questions elicit strength of preference judgments, and do not refer to direct choices or preferences.

Following Lopes' analysis, we assume that the elicited strengths of preferences are represented by differences in PT or CPT values. The change in PT value for the Bimodal prospect is obtained by substituting the PT formula (1) and subtracting. The probability of the $\$ 200$ outcome increases from 0.25 to 0.30 and therefore its decision weight increases $\pi(0.30)-\pi(0.25)$. Similarly, the decision weight of the $-\$ 200$ outcome decreases by $\pi(0.25)-\pi(0.20)$. For all other outcomes the probabilities and decision weights remain unaffected. For the Bimodal prospect, the PT change resulting from the outcome change is therefore

$$
(\pi(0.30)-\pi(0.25)) v(\$ 200)-(\pi(0.25)-\pi(0.20)) v(-\$ 200)
$$

For the Peaked prospect, the change is

$$
(\pi(0.15)-\pi(0.10)) v(\$ 200)-(\pi(0.10)-\pi(0.05)) v(-\$ 200)
$$

PT makes no clear prediction here. If the weighting function is linear in the region [0.05, 0.30] for most subjects, then the two changes give approximately the same improvement for most subjects, and approximately half of the subjects can be expected to prefer the Peaked-change. Assume, as in Exhibit 2, that the weighting function is actually curved upwards in the region $[0.05,0.30]$. Then

(i) $\pi(0.30)-\pi(0.25)>\pi(0.15)-\pi(0.10)$, so that the first term in equation (3) is larger than the first term in equation (4);

(ii) $\pi(0.25)-\pi(0.20)>\pi(0.10)-\pi(0.05)$, so that less (a more negative number) is subtracted in equation (3) than in (4).

Both (i) and (ii) make equation (3) larger than (4). Therefore, PT predicts that a majority of subjects will prefer the Bimodal-change. Experimentally, however, a clear majority $(84 \%$ of the subjects in Lopes' experiment, and $63 \%$ in our replication), preferred the Peaked-change. PT can accommodate these findings if the weighting function is curved downwards in the region [0.05, 0.30], as in Exhibit $3 .{ }^{1}$ Then the above inequalities are reversed, leading to a preference for the Peaked prospect.

The experimental finding can also be explained by CPT, as is demonstrated next. The demonstration is based on the observation that the changes of outcomes for the Peaked prospect are located at more extreme tickets than for the Bimodal prospect. CPT predicts that more decision weight is assigned to extreme outcomes, reflecting diminishing sensitivity. Therefore CPT predicts that the changes for the Peaked prospect induce greater changes in evaluation. Next, we show this mathematically. It is convenient for the subsequent analysis to denote the outcomes for each ticket separately (which is permitted in the CPT notations). Thus, we denote the Peaked prospect here by

$$
(-200,0.05 ;-200,0.05 ;-100,0.05 ; \ldots ; 100,0.05 ; 200,0.05 ; 200,0.05)
$$

and the Bimodal prospect similarly.

In cumulative formulas, it is useful to preserve the rank-ordering of outcomes in the subsequent analysis because then all prospects have the same decision weights. ${ }^{2}$ This can be ensured in the

\footnotetext{
${ }^{1}$ It may be useful here to emphasize that the PT weighting function applies to different probabilities from the one of CPT, i.e. to fixed-outcome probabilities and not to cumulative probabilities.

${ }^{2}$ In general, a prospect $\left(y_{1}, p_{1} ; \ldots ; y_{n}, p_{n}\right)$ with $y_{1} \leqslant \ldots \leqslant y_{k} \leqslant 0 \leqslant y_{k+1} \leqslant \ldots \leqslant y_{n}$ has the same decision weights $\pi_{1}^{-}, \ldots, \pi_{k}^{-}$, $\pi_{k+1}^{+}, \ldots, \pi_{n}^{+}$as a prospect $\left(x_{1}, p_{1} ; \ldots ; x_{n}, p_{n}\right)$ with $x_{1} \leqslant \ldots \leqslant x_{k} \leqslant 0 \leqslant x_{k+1} \leqslant \ldots \leqslant x_{n}$. This can be inferred from formula (2) and the subsequent text.
} 
Peaked Prospect

\begin{tabular}{|c|c|c|c|c|c|c|c|c|c|c|}
\hline Ticket & & $p l$ & step 2 & 3 & $p 4$ & & step 1 & step 2 & step 3 & step 4 \\
\hline 20 & $\$ 200$ & $\$ 200$ & $\$ 200$ & $\$ 200$ & $\$ 200$ & $\$ 200$ & $\$ 200$ & $\$ 200$ & $\$ 200$ & $\$ 200$ \\
\hline 19 & $\$ 200$ & $\$ 200$ & $\$ 200$ & $\$ 200$ & $\$ 200$ & $\$ 200$ & $\$ 200$ & $\$ 200$ & $\$ 200$ & $\$ 200$ \\
\hline 18 & $\$ 100$ & $\$ 100$ & $\$ 100$ & $\$ 100$ & $\$ 200$ & $\$ 200$ & $\$ 200$ & $\$ 200$ & $\$ 200$ & $\$ 200$ \\
\hline 17 & $\$ 100$ & $\$ 100$ & $\$ 100$ & $\$ 100$ & $\$ 100$ & $\$ 200$ & $\$ 200$ & $\$ 200$ & $\$ 200$ & $\$ 200$ \\
\hline 16 & $\$ 100$ & $\$ 100$ & $\$ 100$ & $\$ 100$ & $\$ 100$ & $\$ 200$ & $\$ 200$ & $\$ 200$ & $\$ 200$ & $\$ 200$ \\
\hline 15 & $\$ 100$ & $\$ 100$ & $\$ 100$ & $\$ 100$ & $\$ 100$ & $\$ 100$ & $\$ 100$ & $\$ 100$ & $\$ 100$ & $\$ 200$ \\
\hline 14 & $\$ 0$ & $\$ 0$ & $\$ 0$ & $\$ 100$ & $\$ 100$ & $\$ 100$ & $\$ 100$ & $\$ 100$ & $\$ 100$ & $\$ 100$ \\
\hline 13 & $\$ 0$ & $\$ 0$ & $\$ 0$ & $\$ 0$ & $\$ 0$ & $\$ 100$ & $\$ 100$ & $\$ 100$ & $\$ 100$ & $\$ 100$ \\
\hline 12 & $\$ 0$ & $\$ 0$ & $\$ 0$ & $\$ 0$ & $\$ 0$ & $\$ 100$ & $\$ 100$ & $\$ 100$ & $\$ 100$ & $\$ 100$ \\
\hline 11 & $\$ 0$ & $\$ 0$ & $\$ 0$ & $\$ 0$ & $\$ 0$ & $\$ 0$ & $\$ 0$ & $\$ 0$ & $\underline{\$ 100}$ & $\$ 100$ \\
\hline 10 & $\$ 0$ & $\$ 0$ & $\$ 0$ & $\$ 0$ & $\$ 0$ & $\$ 0$ & $\$ 0$ & $\$ 0$ & $\$ 0$ & $\$ 0$ \\
\hline 9 & $\$ 0$ & $\$ 0$ & $\$ 0$ & $\$ 0$ & $\$ 0$ & $-\$ 100$ & $-\$ 100$ & $\underline{\$ 0}$ & $\$ 0$ & $\$ 0$ \\
\hline 8 & $\$ 0$ & $\$ 0$ & $\$ 0$ & $\$ 0$ & $\$ 0$ & $-\$ 100$ & $-\$ 100$ & $-\$ 100$ & $-\$ 100$ & $\$ 100$ \\
\hline 7 & $\$ 0$ & $\$ 0$ & $\$ 0$ & $\$ 0$ & $\$ 0$ & $-\$ 100$ & $-\$ 100$ & $-\$ 100$ & $-\$ 100$ & $-\$ 100$ \\
\hline 6 & $-\$ 100$ & $-\$ 100$ & $\underline{\$ 0}$ & $\$ 0$ & $\$ 0$ & $-\$ 100$ & $-\$ 100$ & $-\$ 100$ & $-\$ 100$ & $-\$ 100$ \\
\hline 5 & $-\$ 100$ & $-\$ 100$ & $-\$ 100$ & $-\$ 100$ & $-\$ 100$ & $-\$ 200$ & $-\$ 100$ & $-\$ 100$ & $-\$ 100$ & $-\$ 100$ \\
\hline 4 & $-\$ 100$ & $-\$ 100$ & $-\$ 100$ & $-\$ 100$ & $-\$ 100$ & $-\$ 200$ & $-\$ 200$ & $-\$ 200$ & $-\$ 200$ & $-\$ 200$ \\
\hline 3 & $-\$ 100$ & $-\$ 100$ & $-\$ 100$ & $-\$ 100$ & $-\$ 100$ & $-\$ 200$ & $-\$ 200$ & $-\$ 200-$ & $-\$ 200$ & $-\$ 200$ \\
\hline 2 & $-\$ 200$ & $-\$ 100$ & $-\$ 100$ & $-\$ 100$ & $-\$ 100$ & $-\$ 200$ & $-\$ 200$ & $-\$ 200-$ & $-\$ 200$ & $-\$ 200$ \\
\hline 1 & $-\$ 200$ & $-\$ 200$ & $-\$ 200$ & $-\$ 200$ & $-\$ 200$ & $-\$ 200$ & $-\$ 200$ & $-\$ 200-$ & $-\$ 200$ & $-\$ 200$ \\
\hline
\end{tabular}

Bimodal Prospect

step 1 step 2 step 3 step 4 
For the Bimodal prospect, the four changes from $-\$ 200$ to $-\$ 100$, from $-\$ 100$ to $\$ 0$, from $\$ 0$ to $\$ 100$, and from $\$ 100$ to $\$ 200$, are located at tickets $5,9,11$, and 15 , and generate increases in CPT values of $\pi_{5}^{-}(v(-100)-v(-200)), \pi_{9}^{-}(v(0)-v(-100)), \pi_{11}^{+}>(v(100)-v(0))$, and $\pi_{15}^{+}(v(200)-$ $v(100))$. The sum of these four increases is the increase of CPT value generated by changing one $-\$ 200$ ticket into a \$200 ticket for the Bimodal prospect.

The first, second, and fourth increases are clearly greater for the Peaked prospect, because $\pi_{2}^{-}>\pi_{5}^{-}, \pi_{6}^{-}>\pi_{9}^{-}$, and $\pi_{18}^{+}>\pi_{15}^{+}$, assuming the weighting function $w^{+}$of Exhibit 3 . For example, $\pi_{18}^{+}>\pi_{15}^{+}$can be rewritten as $w^{+}(0.15)-w^{+}(0.10)>w^{+}(0.30)-w^{+}(0.25)$, which inequality can be inferred from Exhibit 3. The other inequalities can be derived similarly. The third increase is approximately the same for both prospects. In sum, the overall increase for the Peaked prospect is larger than for the Bimodal prospect. This prediction of CPT is in agreement with the empirical finding.

The above derivation shows that the CPT functional reflects diminishing sensitivity with respect to probability transformations. For the change that is offered in the Peaked prospect, the probability of the most serious loss is reduced from 0.10 to 0.05 , whereas in the Bimodal prospect this probability is reduced from 0.25 to 0.20 . Psychologically, the latter change has less impact. On the gain side, the change offered also favors the Peaked prospect, for people are more sensitive to a change in probability from 0.10 to 0.15 than to a change of 0.25 to 0.30 .

\section{EXPERIMENT 2}

In the second experiment, the different predictions of CPT are a result of the cumulative method of valuation, and the general formula of PT is falsified irrespective of what weighting function is used in PT. We consider the prospect, illustrated in Exhibit 6 , that yields $-\$ 300$ if ticket 1,2 , or 3 is drawn, $\ldots$, and $\$ 300$ if ticket 19,20 , or 21 is drawn. The experiment concerns changes of this prospect obtained by moving one ticket a category upwards. For example, one ticket that yields $\$ 0$ can be changed into a ticket yielding $\$ 100$. Changing a $\$ 300$ outcome is not included, so six changes are possible. Subjects ( $n=42$, undergraduate students) had to choose between two prospects that both resulted from the original prospect by a (different) change of a ticket. For all 15 possible pairs of such altered prospects, subjects had to make a choice. The classical expected utility model with risk aversion (i.e. diminishing marginal utility) predicts that subjects prefer to move tickets associated with lower outcomes. Next, we analyze the predictions of PT and CPT. We shall find the strongest divergence for the preference between changing a $-\$ 300$ outcome and changing a $-\$ 200$ outcome, and for the preference between changing a $\$ 100$ outcome and changing a $\$ 200$ outcome.

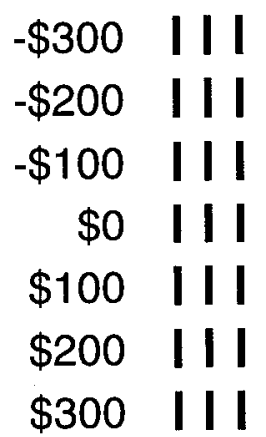

Exhibit 6. Bars indicate lottery tickets, numbered from the upper left to the lower right 
Consider changing $x$ into $x+100$ for one ticket. Such a change is called an ' $x$ move' henceforth. We calculate the resulting PT change. The probability of the outcome $x$ decreases from $3 / 21$ to $2 / 21$, hence its contribution to the PT value decreases by $(\pi(3 / 21)-\pi(2 / 21)) v(x)$. The probability of the outcome $x+100$ increases from $3 / 21$ to $4 / 21$. Hence its contribution to the PT value increases by $(\pi(4 / 21)-$ $\pi(3 / 21)) v(x+100)$. In total, the PT change is

$$
(\pi(4 / 21)-\pi(3 / 21)) v(x+100)-(\pi(3 / 21)-\pi(2 / 21)) v(x)
$$

Subtracting $(\pi(4 / 21)-\pi(3 / 21)) v(x)$ from the first term and adding it to the second gives

$$
(\pi(4 / 21)-\pi(3 / 21))(v(x+100)-v(x))+(\pi(4 / 21)-2 \pi(3 / 21)+\pi(2 / 21)) v(x)
$$

Let us consider the first term in formula (5). This captures the effect of curvature of the value function. For losses, diminishing sensitivity implies that the value difference $v(x+100)-v(x)$ will be largest for $x=-100$, smaller for $x=-200$, and smallest for $x=-300$. For gains, diminishing sensitivity implies that the value difference will be largest for $x=0$, smaller for $x=100$, and smallest for $x=200$. Comparisons between gains and losses cannot be predicted with certainty but, because 'losses loom larger than gains', ${ }^{3}$ the changes for losses will be larger than the changes for the corresponding gains. At any rate, both for gains and for losses, the first term in formula (5) is larger for middle values of $x$ than for extreme values.

Next we consider the second term in formula (5); this captures the effect of curvature of the weighting function. For a subject for whom

$$
\pi(4 / 21)-2 \pi(3 / 21)+\pi(2 / 21) \geqslant 0
$$

(this holds under 'convexity', such as in Exhibit 2), the second term is increasing in $x$, thus is largest for $x=200$, and smallest for $x=-300$. Combining this with the behavior of the first term, we conclude that the change at $x=-200$ then is larger than the change at $x=-300$. A comparison between the changes at $x=100$ and $x=200$ cannot be made directly because the first and second term produce opposite effects.

For a subject for whom

$$
\pi(4 / 21)-2 \pi(3 / 21)+\pi(2 / 21) \leqslant 0
$$

(this holds under 'concavity', such as in Exhibit 3) the second term is decreasing in $x$, thus is largest for $x=-300$, and smallest for $x=200$. Combining this with the behavior of the first term, we conclude that the change at $x=100$ then is larger than the change at $x=200$. Now a comparison between the changes at $x=-200$ and $x=-300$ cannot be made directly, because the first and second terms produce opposite effects.

Let us summarize the predictions of PT concerning two preferences, the preference between the -200 move and -300 move, and the preference between the 100 move and the 200 move.

(1) If formula (6) holds for the majority of subjects,

then -200 move strictly preferred over -300 move for the majority;

and $\quad 100$ move?

(2) If formula (7) holds for the majority of subjects,

then $\quad 100$ move strictly preferred over 200 move for the majority;

and -200 move? $\quad-300$ move (no clear prediction).

\footnotetext{
${ }^{3}$ This phenomenon is confirmed by the experiment, for $69 \%$ of the subjects preferred the -300 move over the 200 move, $67 \%$ preferred the -200 move over the 100 move, and $79 \%$ preferred the -100 move over the 0 move.
} 
If there is no pronounced majority of subjects for whom inequalities (6) or (7) hold, then there will be majority preferences both for the -200 move over the -300 move, and for the 100 move over the 200 move.

Overall, PT predicts that more subjects will prefer moving middle outcomes than moving extreme outcomes. Lopes' experiment gave opposite results: a clear majority $(90 \%)$ preferred the -300 move over the -200 move, and similarly the majority ( $86 \%$ ) preferred the 200 move over the 100 move.

We now turn to an analysis of the above example through CPT, where diminishing sensitivity and thus the weighting function of Exhibit 3 are assumed. We locate the moves at tickets 3, 6, 9, 12, 15, and 18 , respectively, to preserve the rank-ordering where ticket 1 always yields the lowest outcome, ...., and ticket 21 the highest. First, we concentrate on the role of the value function and disregard the effect of the weighting function (i.e. assume it is linear). Then, for losses, diminishing sensitivity of the value function implies that the -100 move is most preferred, the -200 move is less preferred, and the -300 move is least preferred. For gains, diminishing sensitivity implies that the 0 move is most preferred, the 100 move is less preferred, and the 200 move is least preferred.

Next we concentrate on the role of the weighting function, and disregard the effect of the value function. By diminishing sensitivity of the weighting function $w^{-}$for losses, the tickets associated with more extreme outcomes get higher decision weights and the -300 move (ticket 3 ) is preferred to the -200 move (ticket 6 ), which in turn is preferred to the -100 move (ticket 9). Similarly, diminishing sensitivity of the weighting function $w^{+}$implies that the 200 move is preferred to the 100 move, which is preferred to the 0 move.

Summarizing, both for gains and for losses, curvature of the value function favors middle-outcome moves and curvature of the weighting function favors extreme-outcome moves. These effects being opposite, at this stage no definite predictions can be made. To decide on the prediction of CPT, we must decide which effect can be expected to be stronger. The outcomes are not very extreme and span only a small part of the total assets of the subjects (from $-\$ 300$ until $-\$ 100$ and from $\$ 0$ until $\$ 200$, respectively). Hence, the effects of curvature of value are small, and the effects of probability weighting will be stronger. Therefore, a majority preference for the -300 move can be expected over the -200 move, in agreement with the above findings of Lopes' experiment $(86 \%) .{ }^{4}$ Similarly, a majority preference for the 200 move over the 100 move is predicted, again in agreement with the experiment $(90 \%) .^{5}$

In summary, in the second experiment CPT predicts a majority preference for extreme-outcome moves, both for gains and for losses, whereas PT predicts that at least in one case there should be a preference for middle-outcome moves. The great majority of subjects followed the CPT prediction. This result holds independently of the weighting function that one adopts in PT, and therefore this experiment provides empirical evidence against the general form of PT.

\section{CONCLUSION}

This paper has discussed differences between the original prospect theory and the new 'cumulative' prospect theory. The latter has many advantages. Not only does it satisfy stochastic dominance, but

\footnotetext{
${ }^{4}$ The -300 move was preferred over the -100 move by $60 \%$ of the subjects (considerably less than $90 \%$ ). This weaker preference can be explained because the decision weight of the ticket for the -100 move is not much smaller than of the ticket for the -200 move ( $w^{-}$being approximately linear in the interior domain), but the value difference for the -100 move is considerably larger than for the -200 move, $v$ being steepest near 0 . Therefore the value function effect becomes relevant for the -100 move, and the move is less clearly dispreferred to the -300 move. This explains why we considered, for losses, the preference between the -300 and -200 moves in the main text: here the CPT prediction differs most strongly from the PT prediction.

${ }^{5}$ The 200 move was preferred over the 0 move by $69 \%$ of the subjects. The discussion is similar to the previous footnote.
} 
we also find that it gives a better account for a number of empirical findings. Lopes' first experiment provides evidence for an S-shaped weighting function as in cumulative prospect theory, and her second experiment provides evidence against the general formula of prospect theory, independently of the adopted weighting function.

We hope that this paper has shown that the mathematical forms proposed by cumulative prospect theory agree well with the psychological principle of diminishing sensitivity.

\section{ACKNOWLEDGEMENTS}

We thank Lola Lopes for providing us with data from her experiments. We also thank three anonymous referees and Thom Bezembinder for their numerous and highly constructive comments.

\section{REFERENCES}

Allais, M. 'The general theory of random choices in relation to the invariant cardinal utility function and the specific probability function', in Munier, B. R. (ed.), Risk, Decision and Rationality (pp. 233-289), Dordrecht: Reidel, 1988.

Camerer, C. F. 'An experimental test of several generalized utility theories', Journal of Risk and Uncertainty, 2 (1989), 61-104.

Camerer, C. F. 'Recent tests of generalizations of expected utility theory', in Edwards, W. (ed.), Utility Theories: Measurement and Applications (pp. 207-251), Dordrecht: Kluwer Academic Publishers, 1992.

Camerer, C. F. and Ho, T.-H. 'Violations of the betweenness axiom and nonlinearity in probability', Journal of Risk and Uncertainty, 8 (1994), 167-196.

Kahneman, D. and Tversky, A. 'Prospect theory: an analysis of decision under risk', Econometrica, 47 (1979), 263-291.

Lopes, L. L. 'Risk and distributional inequality', Journal of Experimental Psychology: Human Perception and Performance, 10 (1984), 465-485.

Lopes, L. L. 'Reasons and resources: the human side of risk taking', in Bell, N. and Bell, R. (eds), Adolescent Risk Taking, Lubbock, TX: Sage, 1993.

Luce, R. D. 'Rank-dependent, subjective expected-utility representations', Journal of Risk and Uncertainty, 1 (1988), 305-332.

Luce, R. D. and Fishburn, P. C. 'Rank- and sign-dependent linear utility models for finite first-order gambles', Journal of Risk and Uncertainty, 4 (1991), 29-59.

Miyamoto, J. M. 'Constraints on the representation of gambles in prospect theory', Journal of Mathematical Psychology, 31 (1987), 410-418.

Quiggin, J. 'A theory of anticipated utility', Journal of Economic Behaviour and Organization, 3 (1982) 323-343.

Schneider, S. L. and Lopes, L. L. 'Reflection in preferences under risk: who and when may suggest why', Journal of Experimental Psychology: Human Perception and Performance, 12 (1986), 535-548.

Slovic, P., Lichtenstein, S. and Fischhoff, B. 'Decision making', in Atkinson, R. C. et al. (eds), Steven's Handbook of Experimental Psychology, 2nd edition, New York: Wiley, 1988.

Starmer, C. 'Testing new theories of choice under uncertainty using the common consequence effect', Review of Economic Studies, 59 (1992), 813-830.

Starmer, C. and Sugden, R. 'Violations of the independence axiom in common ratio problems: an experimental test of some competing hypotheses', Annals of Operations Research, 19 (1989), 79-101.

Tversky, A. and Kahneman, D. 'Advances in prospect theory: cumulative representation of uncertainty', Journal of Risk and Uncertainty, 5 (1992), 297-323.

Wakker, P. P. 'Transforming probabilities without violating stochastic dominance', in Roskam, E.E.Ch.I. (ed.), Mathematical Psychology in Progress (pp. 29-47), Berlin: Springer, 1989.

Weber, E. U. 'From subjective probabilities to decision weights: the effects of asymmetric loss functions on the evaluation of uncertain outcomes and events', Psychological Bulletin, 115 (1994), 228-242. 


\section{Authors' biographies:}

Hein Fennema obtained a master's degree in philosophy and is now working towards his $\mathrm{PhD}$ in psychology at the NICI institution of the University of Nijmegen. His research interest is in decision under risk/uncertainty.

Peter Wakker is Associate Professor in medical decision making at the University of Leiden. His research interests are in decision under risk/uncertainty and utility measurement.

Authors' addresses:

Hein Fennema, NICI, Mathematical Psychology, University of Nijmegen, PO Box 9104, 6500 HE Nijmegen, The Netherlands.

Peter Wakker, Medical Decision Making Unit, University of Leiden, Leiden, The Netherlands. 